University of Nebraska - Lincoln

DigitalCommons@University of Nebraska - Lincoln

Faculty Publications in Computer \& Electronics Electrical \& Computer Engineering, Department Engineering (to 2015)

9-2009

\title{
Ergodic Capacity of Cooperative Networks using Adaptive Transmission and Selection Combining
}

\author{
Bao Q. Vo-Nguyen \\ University of Ulsan, Korea, baovnq@mail.ulsan.ac.kr \\ Trung Q. Duong \\ Blekinge Institute of Technology, Sweden, quang.trung.duong@bth.se \\ Nguyen N. Tran \\ University of Nebraska-Lincoln, n.n.tran@ieee.org
}

Follow this and additional works at: https://digitalcommons.unl.edu/computerelectronicfacpub

Part of the Computer Engineering Commons

\begin{abstract}
Vo-Nguyen, Bao Q.; Duong, Trung Q.; and Tran, Nguyen N., "Ergodic Capacity of Cooperative Networks using Adaptive Transmission and Selection Combining" (2009). Faculty Publications in Computer \& Electronics Engineering (to 2015). 65.

https://digitalcommons.unl.edu/computerelectronicfacpub/65
\end{abstract}

This Article is brought to you for free and open access by the Electrical \& Computer Engineering, Department of at DigitalCommons@University of Nebraska - Lincoln. It has been accepted for inclusion in Faculty Publications in Computer \& Electronics Engineering (to 2015) by an authorized administrator of DigitalCommons@University of Nebraska - Lincoln. 


\title{
Ergodic Capacity of Cooperative Networks using Adaptive Transmission and Selection Combining
}

\author{
Bao Q. Vo-Nguyen*, Trung Q. Duong ${ }^{\dagger}$, Nguyen. N. Tran ${ }^{\ddagger}$ \\ ${ }^{*}$ School of Electrical Engineering \\ University of Ulsan,San 29 of MuGeo Dong, Nam-Gu, Ulsan, Korea 680-749 \\ E-mail: baovnq@mail.ulsan.ac.kr \\ ${ }^{\dagger}$ The Radio Communications Group \\ Blekinge Institute of Technology, P.O. Box 520, SE-372 25 Ronneby, Sweden \\ E-mail: quang.trung.duong@bth.se \\ $\ddagger$ Department of Computer and Electronics Engineering \\ University of Nebraska-Lincoln, Omaha, NE 68182 USA \\ E-mail: n.n.tran@ieee.org
}

\begin{abstract}
Closed-form expressions for ergodic capacity of repetition-based cooperative networks under adaptive transmission with selection combining are derived. According to the changing channel conditions, the source adapts its rate and/or power level while the relays simply amplify and then forward the received signals. Specifically, three different adaptive techniques are investigated under the assumption of independent Rayleigh fading channels: optimal simultaneous power and rate adaptation (OPRA), constant power with optimal rate adaptation (OPA) and channel inversion with fixed rate (TIFR). Among them, for an arbitrary number of relays, TIFR gives the worst channel capacity; OPRA gives the best channel capacity and ORA has a channel capacity quality in between the others. The analysis results, based on the upper and lower bound of the end-to-end signal-to-noise ratio (SNR), agree very well with the simulated results and definitely show the impact of selection combining on the calculated channel capacity per unit bandwidth.
\end{abstract}

Index Terms - cooperative diversity, adaptive transmission, amplify-and-forward, Shannon capacity, selection combining.

\section{INTRODUCTION}

The concept of cooperative relaying has recently drawn a great attention in the research community due to its potential in improving the reliability of data transmission and in extending coverage of wireless networks in a cost-effective manner (see, e.g. [1], [2] and the references therein). It is based on the broadcast nature of the wireless medium and enables wireless nodes, which are in the communication range of a source, to relay the source information toward the destination. Several cooperation strategies with different relaying techniques, including amplify-and-forward (AF), decode-and-forward (DF), and selective relaying, have been proposed and investigated in terms of outage and bit error probability [3]-[9]. Furthermore, cooperative transmissions have been categorized into spacetime coded cooperation [3], [4], repetition-based cooperation [5]-[7] and opportunistic relay selection cooperation [8], [9].

Recently, optimum resource allocation has emerged as an important research topic to improve the performance of cooperative networks (e.g., see [10], [11]). Particularly, in [10], an opportunistic decode-and-forward protocol is proposed where the relay terminal is utilized depending on the overall network state with dynamic power and time allocation. Furthermore, the effect of partial channel state information at the transmitter (CSIT) on the performance of cooperative communications for delay limited applications is provided. The authors showed that the opportunistic decode-and-forward protocol brings a considerable improvement compared to direct transmission or multi-hop. In [11], the upper bounds and lower bounds on the outage capacity and the ergodic capacity as well as power allocation of three-node wireless relay networks in a Rayleighfading environment are studied when practical constraints on the transmission/reception duplexing at the relay node and on the synchronization between the source node and the relay node is taken into account. Compared to the direct transmission and traditional multihop protocols, the paper reveals that optimum relay channel signaling can significantly outperform multihop protocols, and that power allocation has a significant impact on the performance.

Most of the above-mentioned studies, however, focus on the optimum power allocation with assumption that accurate channel state information (CSI) of all links in the network are available at each nodes. Furthermore, these systems are effectively designed for the worst-case channel conditions, resulting in insufficient utilization of the full channel capacity. This can be ameliorated through the use of adaptive transmission whereby the source adapts its rate and/or power level in response to fading conditions. The idea of applying adaptive transmission for cooperative networks is pioneered by Tyler et. al. in [12] in which the capacity of Rayleigh fading for repetition-based cooperative networks employing maximal ratio combining (MRC) at the destination under three adaptive policies: optimal simultaneous power and rate adaptation, constant power with optimal rate adaptation and channel inversion with fixed rate is provided.

For repetition-based cooperative networks, the destination can employ a variety of diversity combining techniques to obtain diversity from the multiple signal replicas from the 
relays and the source. Although optimum performance is highly desirable, practical systems often sacrifice some performance in order to reduce their complexity. Instead of using MRC, which requires exact knowledge of the channel state information, a repetition-based cooperative system may use selection combining ( $\mathrm{SC}$ ), which is the simplest combining method and can be used in conjunction with differentially coherent and noncoherent modulation techniques [13]-[18] since it does not require knowledge of the signal phases on each branch as would be needed [19]. More specifically, the destination only selects the best signal out of all replicas for further processing and neglects all the remaining ones. This reduces the computational costs and may even lead to a better performance than MRC, because channels with very low signal-to-noise ratio (SNR) cannot be accurately estimated and contribute much noise [20].

In this paper, we provide a capacity analysis of Rayleigh fading channel for AF repetition-based cooperative networks with SC under adaptive transmission by applying the seminal theory developed in [21], [22]. Because of the relatively complicated statistics of AF repetition-based cooperative relaying [23], [24], the probability density function (PDF) of the upper and lower bounds of the end-to-end SNR are derived and then used to evaluate the system capacity.

The remainder of this paper is organized as follows. In Section II, the system model under investigation and the upper and lower bounds of the end-to-end SNR expressed in a tractable form are provided. In section III, the optimum and sub-optimum adaptation policies for AF repetition-based cooperative relaying are studied in terms of Rayleigh fading capacity. Finally, numerical results are given in Section IV and conclusions are drawn in Section V.

\section{SYSTEM MODEL}

Consider a distributed wireless cooperative network in which the source $(S)$ communicates with the destination (D) with the help of $N$ relay nodes, denoted as $\mathrm{R}_{1} \cdots \mathrm{R}_{i} \cdots \mathrm{R}_{N}$ using amplify-and-forward relaying. Each node is equipped with single antenna and operates in half-duplex mode. All transmissions are assumed orthogonal either in time or in frequency. To facilitate the explanation, we assume a timedivision protocol with $N+1$ time slots.

We denote the source-to-destination, source-to-the $i$-th relay and the $i$-th relay-to-destination link coefficient by $h_{\mathrm{SD}}, h_{\mathrm{SR}_{\mathrm{i}}}$ and $h_{\mathrm{R}_{i} \mathrm{D}}$, respectively. Due to Rayleigh fading, $h_{\mathrm{SD}}, h_{\mathrm{SR}_{i}}$ and $h_{\mathrm{R}_{i} \mathrm{D}}$ are statistically modeled as zero-mean, independent, circularly symmetric, complex Gaussian random variables with variances $\lambda_{0}, \lambda_{1, i}$ and $\lambda_{2, i}$, respectively.

The cooperative transmission under consideration takes places into two phases. In the first phase, the source broadcasts its symbol $s$ with an average transmitted power $\mathcal{P}$ to all the relays and the destination. Under repetition-based AF relaying fashion, the $i$-th relay retransmits the scaled version of the received signal towards the destination in time slot $i+1$ with the amplification factor $\mathcal{G}_{i}$. Mathematically, the system model can be described by the following set of equations as follows:

$$
\begin{gathered}
r_{\mathrm{SD}}=\sqrt{\mathcal{P}} h_{\mathrm{SD}} s+n_{\mathrm{SD}} \\
r_{\mathrm{SR}_{i}}=\sqrt{\mathcal{P}} h_{\mathrm{SR}_{i}} s+n_{\mathrm{SR}_{i}} \\
r_{\mathrm{R}_{i} \mathrm{D}}=\sqrt{\mathcal{P}} h_{\mathrm{R}_{i} \mathrm{D}} \mathcal{G}_{i} r_{\mathrm{SR}_{i}}+n_{\mathrm{R}_{i} \mathrm{D}}
\end{gathered}
$$

where $r_{\mathcal{A B}}=r_{\mathcal{A} \rightarrow \mathcal{B}}$ denotes the received signal at $\mathcal{B}$ sent from $\mathcal{A}$ with $\mathcal{A} \in\left\{\mathrm{S}, \mathrm{R}_{i}\right\}$ and $\mathcal{B} \in\left\{\mathrm{D}, \mathrm{R}_{i}\right\} ; n_{\mathcal{A B}}$ is the additive noise sample at the reception node $\mathcal{B}$ which is modeled as zero-mean mutually independent, circularlysymmetric, complex Gaussian random variable with variance $\mathcal{N}_{0}$. Furthermore, the amplification factor $\mathcal{G}_{i}$ can be defined as

$$
\mathcal{G}_{i}=\sqrt{\frac{1}{\mathcal{P}\left|h_{\mathrm{SR}_{\mathrm{i}}}\right|^{2}+\mathcal{N}_{0}}}
$$

Let us define the effective instantaneous signal-to-noise ratio for $\mathrm{S} \rightarrow \mathrm{D}, \mathrm{S} \rightarrow \mathrm{R}_{i}$ and $\mathrm{R}_{i} \rightarrow \mathrm{D}$ links as $\gamma_{0}=\mathcal{P}\left|h_{\mathrm{SD}}\right|^{2}$, $\gamma_{1, i}=\mathcal{P}\left|h_{\mathrm{SR}_{i}}\right|^{2}$ and $\gamma_{2, i}=\mathcal{P}\left|h_{\mathrm{R}_{i} \mathrm{D}}\right|^{2}$, respectively. Assuming selection combining at the destination, the total combined instantaneous signal-to-noise at the output of the selection combiner, $\gamma_{\Sigma}$, is written by

$$
\gamma_{\Sigma}=\max _{i=0, \ldots, N} \gamma_{i}
$$

where $\left\{\gamma_{i}\right\}_{i=1}^{N}$ can be shown to be [23], [24]

$$
\gamma_{i}=\frac{\gamma_{1, i} \gamma_{2, i}}{\gamma_{1, i}+\gamma_{2, i}+1} \approx \frac{\gamma_{1, i} \gamma_{2, i}}{\gamma_{1, i}+\gamma_{2, i}}
$$

It is hard to derive the exact PDF of (4), therefore, for tractable analysis, (4) can be upper- and lower-bounded as follows [24], [25]:

$$
\frac{1}{2} \min \left(\gamma_{1, i}, \gamma_{2, i}\right) \triangleq \gamma_{i}^{L} \leq \gamma_{i}<\gamma_{i}^{U} \triangleq \min \left(\gamma_{1, i}, \gamma_{2, i}\right)
$$

Since $\gamma_{1, i}$ and $\gamma_{2, i}$ are exponentially distributed random variables with hazard rates $\mu_{1, i}=1 / \bar{\gamma}_{1, i}=1 /\left(\mathcal{P} \lambda_{1, i}\right)$ and $\mu_{2, i}=1 / \bar{\gamma}_{2, i}=1 /\left(\mathcal{P} \lambda_{2, i}\right)$, respectively. Making use the fact that the minimum of two independent exponential random variables is again an exponential random variable with a hazard rate equals to the sum of the two hazard rates [26], i.e., $\mu_{i}=\mu_{1, i}+\mu_{2, i}=\frac{\bar{\gamma}_{1, i}+\bar{\gamma}_{2, i}}{\bar{\gamma}_{1, i} \bar{\gamma}_{2, i}}$. For brevity, by introducing $\bar{\gamma}_{i}=\frac{\mathcal{K}}{\mu_{i}}=\mathcal{K} \frac{\bar{\gamma}_{1, i} \bar{\gamma}_{2, i}}{\bar{\gamma}_{1, i}+\bar{\gamma}_{2, i}}$ and from (5), we have

$$
\begin{gathered}
f_{\gamma_{i}}(\gamma)=\frac{1}{\bar{\gamma}_{i}} e^{-\gamma / \bar{\gamma}_{i}} \\
F_{\gamma_{i}}(\gamma)=\int_{0}^{\gamma} f_{\gamma_{i}}(\gamma) d \gamma=1-e^{-\gamma / \bar{\gamma}_{i}}
\end{gathered}
$$

where $\mathcal{K}$ equals 1 or $1 / 2$ associated with the cases of upper bound and lower bound in (5), respectively. Under the assumption that all links are subject to independent fading, order 
statistics gives the cumulative distribution function (CDF) of $\gamma_{\Sigma}$ as:

$$
F_{\gamma_{\Sigma}}(\gamma)=\operatorname{Pr}\left(\gamma_{0}<\gamma, \ldots, \gamma_{N}<\gamma\right)=\prod_{i=0}^{N} F_{\gamma_{i}}(\gamma)
$$

The joint PDF of $\gamma_{\Sigma}$ is given by differentiating (7) with respect to $\gamma$.

$$
f_{\gamma_{\Sigma}}(\gamma)=\frac{\partial F_{\gamma_{\Sigma}}(\gamma)}{\partial \gamma}=\sum_{i=0}^{N}\left[f_{\gamma_{i}}(\gamma) \prod_{\substack{j=0 \\ j \neq i}}^{N} F_{\gamma_{j}}(\gamma)\right]
$$

Substituting (6a) and (6b) into (8) and after some manipulation yields [27]

$$
\begin{aligned}
f_{\gamma_{\Sigma}}(\gamma) & =\sum_{i=0}^{N}\left[\frac{1}{\bar{\gamma}_{i}} e^{-\frac{\gamma}{\gamma_{i}}} \prod_{\substack{j=0 \\
j \neq i}}^{N}\left(1-e^{-\frac{\gamma}{\gamma_{j}}}\right)\right] \\
& =\sum_{i=0}^{N}(-1)^{i} \sum_{\substack{n_{0}, \ldots, n_{i}=0 \\
n_{0}<\cdots<n_{i}}}^{N} \frac{1}{\chi_{i}} e^{-\frac{\gamma}{\chi_{i}}}
\end{aligned}
$$

where $\chi_{i}=\left(\sum_{l=0}^{i} \bar{\gamma}_{n_{l}}^{-1}\right)^{-1}$.

\section{CAPACITY ANALYSIS}

\section{A. Optimal Simultaneous Power and Rate Adaptation}

Since the equivalent end-to-end CSI $\gamma_{\Sigma}$ is assumed to be known at the source and destination, it allows the source to adapt both its power and rate according to the actual fading channel gain. In particular, the channel capacity of opportunistic cooperative networks over fading channels is given by Goldsmith and Varaiya [21] as

$$
\mathcal{C}_{\text {opra }}=\frac{B}{N+1} \int_{\gamma_{c}}^{+\infty} \log _{2}\left(\frac{\gamma}{\gamma_{c}}\right) f_{\gamma_{\Sigma}}(\gamma) d \gamma
$$

where $B$ is the channel bandwidth in $\mathrm{Hz}$ and $\gamma_{c}$ is the optimal cutoff SNR threshold below which data transmission over the network is halted. The ratio $1 /(N+1)$ in $(10)$ is included to reflect that the source-to-destination information transmission via relays will occupy $N+1$ time slots. The cutoff threshold $\gamma_{c}$ can be determined by using the power constraint [22, eq. 8], namely

$$
\int_{\gamma_{c}}^{+\infty}\left(\frac{1}{\gamma_{c}}-\frac{1}{\gamma}\right) f_{\gamma_{\Sigma}}(\gamma) d \gamma=1
$$

Inserting (9) into (11) leads to

$$
\sum_{i=0}^{N}(-1)^{i} \sum_{\substack{n_{0}, \ldots, n_{i}=0 \\ n_{0}<\cdots<n_{i}}}^{N}\left[\frac{\exp \left(\gamma_{c} / \chi_{i}\right)}{\gamma_{c}}-\frac{E_{1}\left(\gamma_{c} / \chi_{i}\right)}{\chi_{i}}\right]-1=0
$$

where $E_{n}(x)$ is the exponential integral of order $n$ defined by $E_{n}(x)=\int_{1}^{+\infty} t^{-n} e^{-x t} d t, x>0$. With the current form of (12), apparently, the optimal cutoff threshold $\gamma_{c}$ cannot be expressed in an explicit closed form but, in general, can be found by numerically solving (12) ${ }^{1}$.

Next, substituting (9) into (10), we obtain the capacity of AF repetition-based cooperative systems with $\mathrm{SC}$ in terms of the integral $\mathcal{J}_{n}(\mu)=\int_{1}^{+\infty} t^{n-1} \ln t e^{-\mu t} d t[22$, eq. (70)] as follows:

$$
\mathcal{C}_{\text {opra }}=\frac{B}{(N+1) \ln 2} \sum_{i=1}^{N}(-1)^{i} \sum_{\substack{n_{0}, \ldots, n_{i}=1 \\ n_{0}<\cdots<n_{i}}}^{N} \frac{\gamma_{c}}{\chi_{i}} \mathcal{J}_{1}\left(\frac{\gamma_{c}}{\chi_{i}}\right)
$$

According the operation mode of optimal simultaneous power and rate adaptation, the system will stop transmitting when $\gamma_{\Sigma}<\gamma_{c}$. As a result, the system suffers an outage probability, namely

$$
\begin{aligned}
P_{o} & =\operatorname{Pr}\left(\gamma_{\Sigma}<\gamma_{c}\right)=\int_{0}^{\gamma_{c}} f_{\gamma_{\Sigma}}(\gamma) d \gamma \\
& =\sum_{i=0}^{N}(-1)^{i} \sum_{\substack{n_{0}, \ldots, n_{i}=0 \\
n_{0}<\cdots<n_{i}}}^{N}\left(1-e^{-\frac{\gamma_{c}}{\chi_{i}}}\right)
\end{aligned}
$$

\section{B. Optimal Rate Adaptation with Constant Transmit Power}

Since selection combiner is used at the destination, the channel capacity under the condition of optimal rate adaptation with constant transmit power over Rayleigh fading channels is given by [22, eq. (29)]

$$
\begin{aligned}
C_{\text {ora }} & =\frac{B}{N+1} \int_{0}^{+\infty} \log _{2}(1+\gamma) f_{\gamma_{\Sigma}}(\gamma) d \gamma \\
& =\frac{B}{(N+1) \ln 2} \sum_{i=0}^{N}(-1)^{i} \sum_{\substack{n_{0}, \ldots, n_{i}=1 \\
n_{0}<\cdots<n_{i}}}^{N} e^{1 / \chi_{i}} E_{1}\left(1 / \chi_{i}\right)
\end{aligned}
$$

\section{Channel Inversion with Fixed Rate}

In order to maintain a constant received SNR at the destination, the source adapts its transmit power based on the channel fading. As previously discussed in [21], [22], [28], the power adaptation is commonly used and the related channel capacity is given by

$$
\mathcal{C}_{t i f r}=\frac{B}{N+1} \log _{2}\left(1+\frac{1}{\int_{0}^{+\infty} \frac{f_{\gamma_{\Sigma}}(\gamma)}{\gamma} d \gamma}\right)
$$

For total channel inversion, a large amount of the transmitted power is used to compensate for the deep channel fades resulting in a large capacity loss compared with others adaptation techniques. To achieve a better capacity, a modified inversion policy in which the channel is compensated since the

\footnotetext{
${ }^{1}$ It can be done with the help of Matlab function fzero in the optimization toolbox
} 


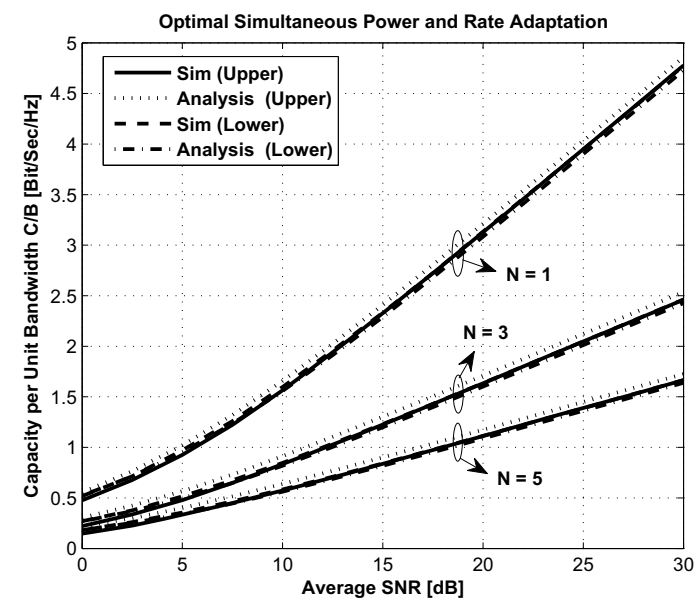

Fig. 1. Channel capacity per unit bandwidth for AF repetition-based cooperative networks with SC under OPRA over i.i.d. channels.

channel fading is greater than a threshold is proposed leading to

$$
\begin{aligned}
\mathcal{C}_{t i f r} & =\frac{B}{N+1} \log _{2}\left(1+\frac{1}{\int_{\gamma_{c}}^{+\infty} \frac{f_{\gamma_{\Sigma}(\gamma)}}{\gamma} d \gamma}\right)\left(1-P_{o}\right) \\
& =\frac{B}{N+1} \log _{2}\left(1+\frac{1}{\sum_{i=0}^{N}(-1)^{i} \sum_{\substack{n_{0}, \ldots, n_{i}=0 \\
n_{0}<\cdots<n_{i}}}^{N} \frac{E_{1}\left(\gamma_{c} / x_{i}\right)}{\chi_{i}}}\right)\left(1-P_{o}\right)
\end{aligned}
$$

where $P_{o}$ has the same form as in (14). However, the optimal cutoff threshold $\gamma_{c}$ here can be selected to satisfy the desired outage probability or alternatively to maximize $\mathcal{C}_{\text {tifr }}$ given in (17). For the latter case, the optimal cutoff threshold can be numerically found with the help of Matlab function fminbnd in the optimization toolbox.

\section{NUMERICAL RESULTS AND DISCUSSION}

In this section, based on the mathematical formalism above, we illustrate the selected analytical and simulation results with $\lambda_{0}=\left\{\lambda_{1, i}\right\}_{i=1}^{N}=\left\{\lambda_{2, i}\right\}_{i=1}^{N}=1$ for independent and identically distributed (i.i.d.) Rayleigh channels and $\lambda_{0}=1$, $\left\{\lambda_{1, i}\right\}_{i=1}^{N}=2$ and $\left\{\lambda_{2, i}\right\}_{i=1}^{N}=3$ for independent but nonidentically distributed (i.n.d.) Rayleigh channels. Furthermore, performance results are reported in terms of the capacity per unit bandwidth versus average SNR in $\mathrm{dB}$.

In Fig. 1 and 2, we present the normalized system capacity over i.i.d. and i.n.d. Rayleigh fading under optimal simultaneous power and rate adaptation. One can see that an increase in the number of relays significantly affects the system capacity. Specifically, it can be observed from Fig. 1 that a transition from $N=1$ to $N=3$ leads to a normalized capacity decrement around $20 \mathrm{~dB}$ at average SNR of $10 \mathrm{~dB}$. Moreover, one may notice that there is a small gap between

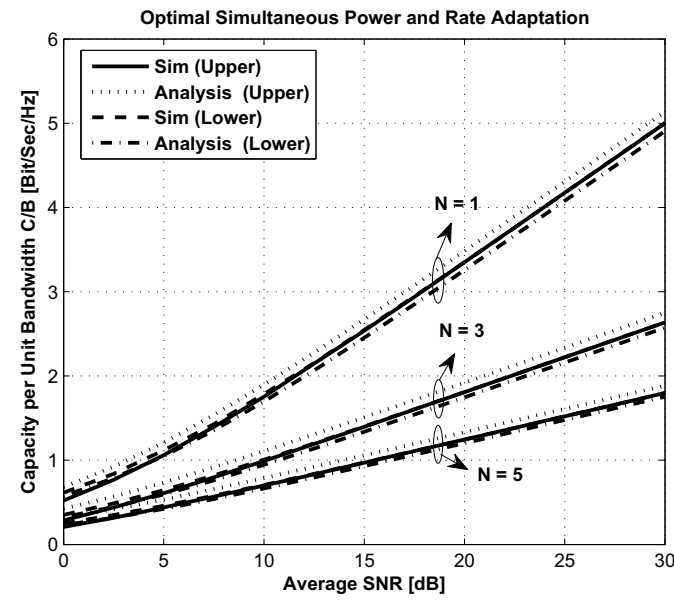

Fig. 2. Channel capacity per unit bandwidth for AF repetition-based cooperative networks with SC under OPRA over i.n.d. channels.

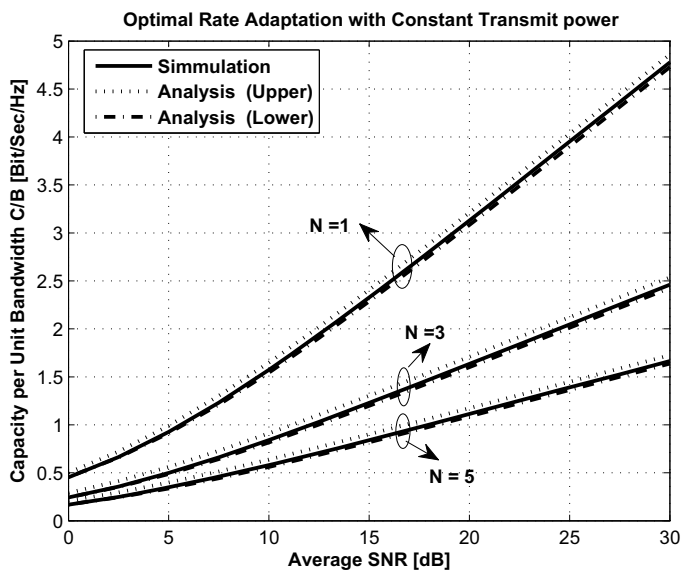

Fig. 3. Channel capacity per unit bandwidth for $\mathrm{AF}$ repetition-based cooperative networks with SC under ORA over i.i.d. channels.

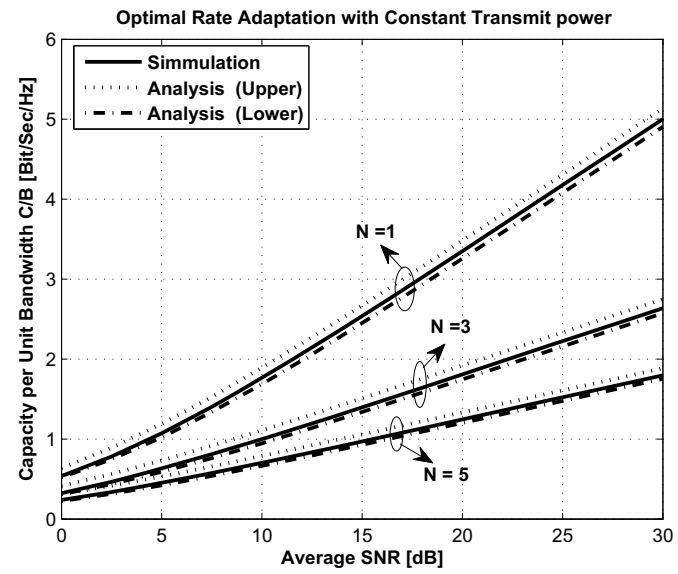

Fig. 4. Channel capacity per unit bandwidth for AF repetition-based cooperative networks with SC under ORA over i.n.d. channels.

the upper and lower analytical bound, which can be obtained 


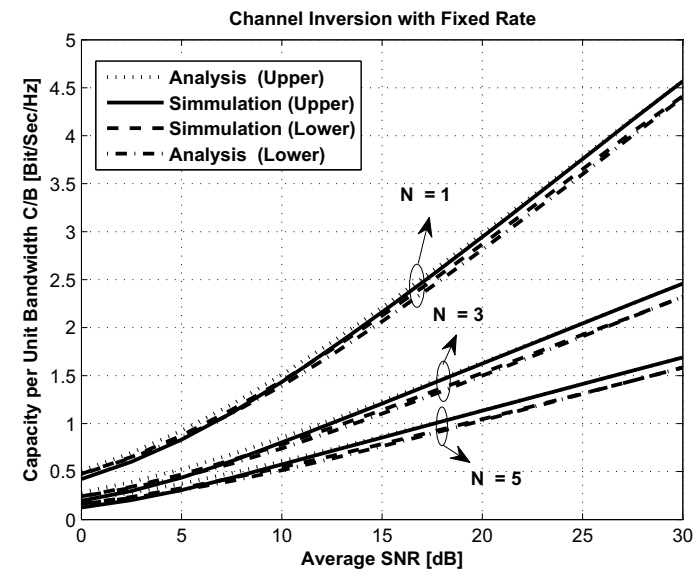

Fig. 5. Channel capacity per unit bandwidth for AF repetition-based cooperative networks with SC under TIFR over i.i.d. channels.

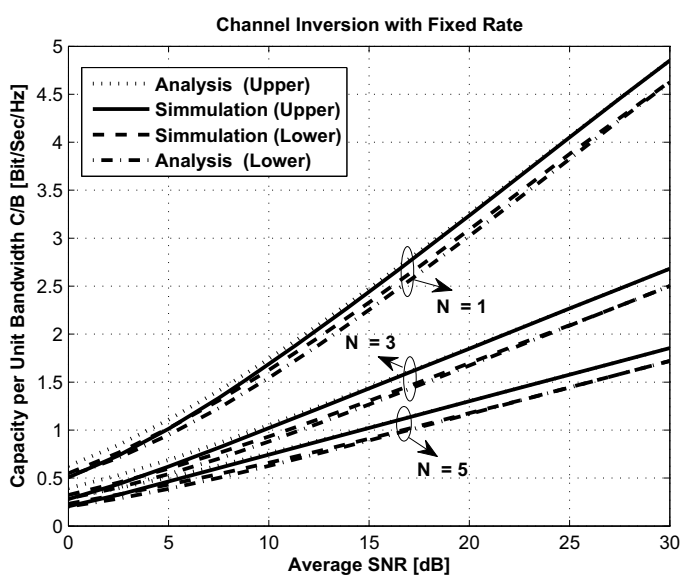

Fig. 6. Channel capacity per unit bandwidth for AF repetition-based cooperative networks with SC under TIFR over i.n.d. channels.

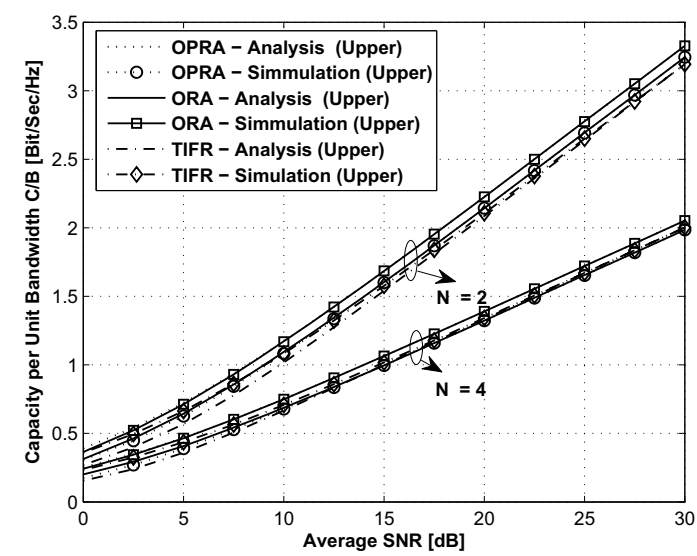

Fig. 7. Channel capacity per unit bandwidth for AF repetition-based cooperative networks with SC under different adaptation policies over i.n.d. channels.

by adjusting the parameter $\mathcal{K}$. This fact was also noticed in

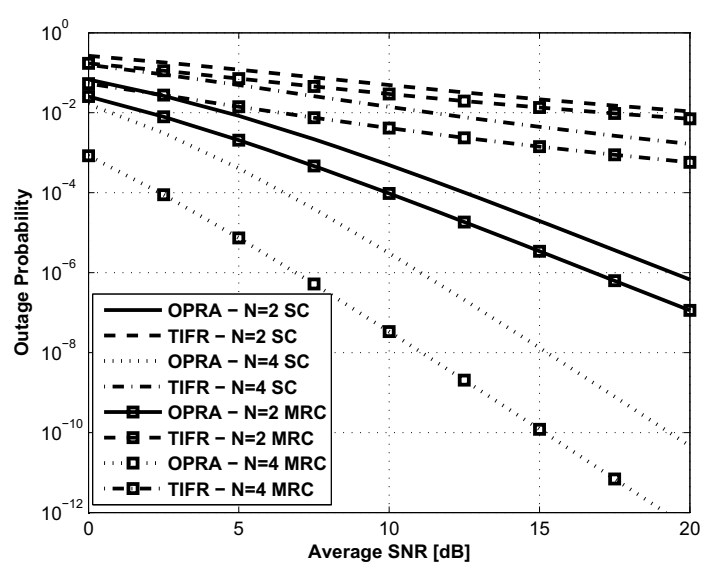

Fig. 8. Outage probability of the ORPA and TIFR for AF repetition-based cooperative networks with SC over i.n.d. channels.

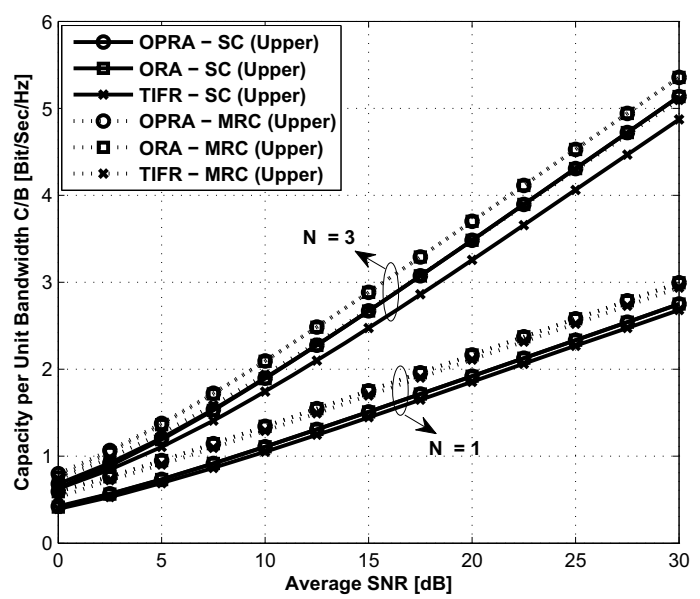

Fig. 9. Comparison of channel capacity per unit bandwidth for AF repetitionbased cooperative networks with MRC and SC under different adaptation policies over i.n.d. channels.

[24] for the amplify-and-forward relaying case undergoing Rayleigh fading. However, for the upper and lower simulated curves, this gap is negligible.

Figs. 3 and 4 depict the channel capacity per unit bandwidth against the average SNR in $\mathrm{dB}$ for optimal rate adaptation with constant transmit power over i.i.d. and i.n.d. channels, respectively with an aim to examine the effects of the number of relays participating in the cooperative transmission as well as to validate analytical results. As expected, increasing $N$ yields degradation in system capacity. Regarding the influence of $N$ on the overall system capacity, note that the normalized capacity obtained from increasing $N$ from 1 to 5 decreases, but with diminishing returns. Furthermore, the analysis results agree very well with the simulation results.

Figs. 5 and 6 show the system normalized-capacity under the channel inversion with fixed rate policy with respect to the optimal cutoff threshold over i.i.d. and i.n.d. Rayleigh fading channels, respectively. It can be clearly seen that a 
similar observation relative other policies can be made when the optimal cutoff is chosen to maximize the system capacity.

In Fig. 7, the closed-form channel capacity given in (13), (15), and (17) are presented and compared over i.n.d. Rayleigh fading with $\mathcal{K}=1$, i.e., using the upper bound. Among them, OPRA provides the best capacity and TIFR provides the worst one, as expected. Fig. 7 also shows that our analytical results are in good agreement with our simulation results. The corresponding outage probability for the optimum adaptation and truncated channel inversion policies are plotted in Fig. 8. As we can see, the benefit of the maximization of the capacity for TIFR systems takes places at the cost of increasing probability of outage.

To investigate the capacity loss due to the use of SC relative to MRC, we plot the calculated channel capacity per unit bandwidth as a function of average SNR for different adaption policies with SC and MRC in Fig. 9. It can be observed from the standpoint of average SNR that the power efficiency of the SC-based networks suffers around 2-dB loss with respect to that of the MRC cooperative networks. Stated another way, curves of normalized capacity for the SC schemes would be parallel to those for the MRC schemes and seem to be shifted $3 \mathrm{~dB}$ to the right.

\section{CONCLUSION}

The paper has investigated and compared AF repetitionbased cooperative networks with selection combining under three different adaptive transmission policies, namely optimal simultaneous power and rate adaptation, constant power with optimal rate adaptation and channel inversion with fixed rate. The analysis is valid and applicable for general cases, including independent identically distributed and independent but not identically distributed Rayleigh fading channels. Numerical results show that by taking advantage of the "timevarying" nature of the wireless environment, the performance of repetition-based cooperative networks is improved. However, the advantage of these schemes comes at the expense of additional hardware complexity to implement adaptive transmission.

\section{REFERENCES}

[1] J. N. Laneman, D. N. C. Tse, and G. W. Wornell, "Cooperative diversity in wireless networks: Efficient protocols and outage behavior," IEEE Transactions on Information Theory, vol. 50, pp. 3062-3080, 2004.

[2] A. Nosratinia, T. E. Hunter, and A. Hedayat, "Cooperative communication in wireless networks," IEEE Communications Magazine, vol. 42, pp. 74-80, 2004.

[3] G. Scutari and S. Barbarossa, "Distributed space-time coding for regenerative relay networks," IEEE Transactions on Wireless Communications, vol. 4, pp. 2387-2399, 2005.

[4] J. N. Laneman and G. W. Wornell, "Distributed space-time coded protocols for exploiting cooperative diversity in wireless networks," in IEEE Global Telecommunications Conference, 2002, 2002, pp. 77-81 vol.1.

[5] N. C. Beaulieu and J. Hu, "A Closed-Form Expression for the Outage Probability of Decode-and-Forward Relaying in Dissimilar Rayleigh Fading Channels," IEEE Communications Letters, vol. 10, pp. 813-815, December 2006.
[6] I. H. Lee and D. Kim, "BER Analysis for Decode-and-Forward Relaying in Dissimilar Rayleigh Fading Channels," IEEE Communications Letters, vol. 11, pp. 52-54, 2007.

[7] F. Xu, F. C. M. Lau, Q. F. Zhou, and D. W. Yue, "Outage Performance of Cooperative Communication Systems Using Opportunistic Relaying and Selection Combining Receiver," IEEE Signal Processing Letters, vol. 16, pp. 113-116, 2009.

[8] A. Bletsas, A. Khisti, D. P. Reed, and A. Lippman, "A Simple Cooperative Diversity Method Based on Network Path Selection," IEEE Journal on Select Areas in Communications, vol. 24, pp. 659-672, March 2006.

[9] A. Bletsas, H. Shin, and M. Z. Win, "Cooperative Communications with Outage-Optimal Opportunistic Relaying," IEEE Transactions on Wireless Communications, vol. 6, pp. 3450-3460, September 2007.

[10] D. Gunduz and E. Erkip, "Opportunistic cooperation by dynamic resource allocation," IEEE Transactions on Wireless Communications, vol. 6, pp. 1446-1454, 2007.

[11] A. Host-Madsen and J. Zhang, "Capacity bounds and power allocation for wireless relay channels," IEEE Transactions on Information Theory, vol. 51, pp. 2020-2040, 2005.

[12] T. Nechiporenko, K. T. Phan, C. Tellambura, and H. H. Nguyen, "On the capacity of Rayleigh fading cooperative systems under adaptive transmission," IEEE Transactions on Wireless Communications, vol. 8, pp. 1626-1631, 2009.

[13] L. Chu, J. Yuan, Y. Li, and Z. Chen, "Differential Modulation and Selective Combining for Multiple-Relay Networks," in IEEE International Conference on Communications Workshops, 2008.

[14] T. Himsoon, W. Pam Siriwongpairat, S. Weifeng, and K. J. R. Liu, "Differential Modulations for Multinode Cooperative Communications," IEEE Transactions on Signal Processing, vol. 56, pp. 2941-2956, 2008.

[15] Q. Zhao and H. Li, "Differential Modulation for Cooperative Wireless Systems," IEEE Transactions on Signal Processing, vol. 55, pp. 22732283, May 2007.

[16] H. Thanongsak, W. P. Siriwongpairat, S. Weifeng, and K. J. R. Liu, "Differential Modulation With Threshold-Based Decision Combining for Cooperative Communications," IEEE Transactions on Signal Processing, vol. 55, pp. 3905-3923, 2007.

[17] Q. Zhao and H. Li, "Performance of differential modulation with wireless relays in Rayleigh fading channels," IEEE Communications Letters, vol. 9, pp. 343-345, 2005.

[18] P. Tarasak, M. Hlaing, and V. K. Bhargava, "Differential modulation for two-user cooperative diversity systems," IEEE Journal on Selected Areas in Communications, vol. 23, pp. 1891-1900, 2005.

[19] J. G. Proakis, Digital communications, 4th ed. Boston: McGraw-Hill, 2001.

[20] V. K*hn, Wireless communications over MIMO channels : applications to CDMA and multiple antenna systems. Chichester, England: John Wiley \& Sons, 2006.

[21] A. J. Goldsmith and P. P. Varaiya, "Capacity of fading channels with channel side information," IEEE Transactions on Information Theory, vol. 43, pp. 1986-1992, 1997.

[22] M. S. Alouini and A. J. Goldsmith, "Capacity of Rayleigh fading channels under different adaptive transmission and diversity-combining techniques," IEEE Transactions on Vehicular Technology, vol. 48, pp. 1165-1181, 1999.

[23] B. Barua, H. Q. Ngo, and H. Shin, "On the SEP of Cooperative Diversity with Opportunistic Relaying," IEEE Communications Letters, vol. 12, pp. 727-729, 2008.

[24] P. A. Anghel and M. Kaveh, "Exact Symbol Error Probability of a Cooperative Network in a Rayleigh-Fading Environment," IEEE Transactions on Wireless Communications, vol. 3, pp. 1416-1421, September 2004.

[25] S. Ikki and M. H. Ahmed, "Performance Analysis of Cooperative Diversity Wireless Networks over Nakagami-m Fading Channel," IEEE Communications Letters, vol. 11, pp. 334-336, 2007.

[26] A. Papoulis and S. U. Pillai, Probability, random variables, and stochastic processes, 4th ed. Boston: McGraw-Hill, 2002.

[27] V. N. Q. Bao, H. Y. Kong, and S. W. Hong, "Performance Analysis of MPAM and M-QAM with Selection Combining in Independent but NonIdentically Distributed Rayleigh Fading Paths," in Vehicular Technology Conference, 2008. VTC 2008-Fall. IEEE 68th, 2008, pp. 1-5.

[28] A. J. Goldsmith and C. Soon-Ghee, "Variable-rate variable-power MQAM for fading channels," IEEE Transactions on Communications, vol. 45 , pp. 1218-1230, 1997. 